\title{
Automated unit for separation of arsenic by iron doped calcium alginate
}

\section{Z. Szücs}

Institute for Nuclear Research of the Hungarian Academy of Sciences, Debrecen, Bem tér 18/C, Hungary

\author{
B. Kovács \\ University Debrecen, Hungary
}

Z. Oláh \& R. Dóczi

Technical University, Budapest, Hungary

M. Maiti

Rorkee University, Rorkee, India

S. Lahiri

Saha Institute of Nuclear Physics, Kolkata, India

ABSTRACT: The prototype of instrument was planned and under the developing for separation of arsenic from drinking water. The highly iron doped calcium alginate is the chemical agent to keep the arsenic species (anions in III and V oxidation stage) on the gel column. The objective is to enrich the capability of production at a concentration of $10 \mu \mathrm{g} \mathrm{L}^{-1} \operatorname{arsenic}$ in $1 \mathrm{~m}^{3}$ of purified water with cost less than 4 USD. During the developing ${ }^{77}$ As and ${ }^{75}$ Se radioisotopes traced the separation. The PLC automated system unit can communicate even trough satellite, and the alginate waste satisfies the "green chemistry" terminology.

\section{INTRODUCTION}

The high concentration of toxic and carcinogen arsenic in drinking water is still the one of the hottest task in Hungary as well as in India. However several technologies are developed for decreasing of the concentration level in $10 \mathrm{mg} \mathrm{m}^{-3}$ (EU normative), they are expensive or create a new danger for environment as waste (Fe precipitation). On the basis of scientific reports of Banerjee et al. (2007), the highly iron doped calcium alginate was chosen for the separation of arsenic from the drinking water due to its high efficiency of retain the arsenic species (anions in III and V oxidation stage). The industrial preparation of the modified alginate gel is costfriendly. The amount of the dried waste is only 2 $3 \%$ of the mass of working gel and not required any additional treatment furthermore. Therefore the alginate gel is a classical "green chemical". This paper discusses about the development of a semi-industrial scale equipment using the above mentioned chemicals for systematic evaluation of parameters to build up an industrial size apparatus producing Ascleaned, cheap drinking water.

\section{METHODS/EXPERIMENTAL}

\subsection{Chemical part}

The preparation of the highly iron doped calcium alginate was published by Banerjee, where the alginate gel contains $\sim 3,8 \%$ of ferri ion, approximately. Lahiri et al. investigated the speciation dependents of removal of arsenic studying the reaction time, $\mathrm{pH}$, arsenic concentration and desorption behaviour.
Their perspective results suggest using the modified alginate in the automated, mobile equipment to be developed for elimination of arsenic. The radioactive tracers, ${ }^{77}$ As and ${ }^{75} \mathrm{Se}$ will be used for studying of the developed system and to trace the arsenic and selenium. The dramatic low level of selenium in drinking water in Hungary required checking the concentration of selenium, because the alginate gel shows affinities for selenium too. Therefore the planned apparatus will contain a selenite adjuster after the elimination of the arsenic. Before the design of the equipment, the whole chemical process will be simulated and checked in laboratory scale.

\subsection{Design of equipment}

The equipment operates in batch and semi-batch mode depending on the amount of the ordered cleaned water. The system will be built up with three main parts. The first part will adjust the $\mathrm{pH}$ of water up to value 3 , where is the most effective the retention of selenate on the gel column. The second part is the cascade of the alginate filled column, where the number of the cascade depends on from the concentration of the arsenate and the amount of inlet water. The third part will readjust the $\mathrm{pH}$ to 7 and add the water soluble selenit. The level of the arsenite in drinking water is lower with 1-2 magnitude than that of arsenate. Therefore usually the removing of arsenate can fulfil the required $10 \mathrm{mg} \mathrm{m}^{-3}$ limit. However, after the $\mathrm{pH}$-readjusting additional alginate gel column unit can be connected to decrease the concentration of arsenite.
Megjegyzés [T1]: The reference must be cited by the first author 


\subsection{Automation and communication}

The equipment will be automated by PLC produced by VACO company. The system automatically adjust the $\mathrm{pH}$ of the inlet water, pump the water through the adequate number of the column uni filled with modified alginate gel. The flow meters, the liquid level sensors, $\mathrm{pH}$-meters will generate the remote signals for the system. One-touch screen helps to check the running parameters as well as the system is able to communicate through the satellite too. In that case a grid of the water treating units can be created covering the full country and to be continually up to date.

\section{RESULTS AND DISCUSSION}

\subsection{Chemical processes}

The highly iron doped calcium alginate shows much more efficiency to remove of arsenate from the water than it was explained in US patent of Min \& Hering (1998). The alginate gel with the $3,8 \%$ of iron content successfully remove the arsenic even from $20 \mathrm{~g} \mathrm{~m}^{-3}$ concentration. The necessary contact time with the gel is also reduced from 12 hs to 30 min by this modified alginate. The trace radioactive isotopes, ${ }^{77} \mathrm{As}$ and ${ }^{75} \mathrm{Se}$, help to monitoring the efficiency of the system in situ and online. The saturated gel contains arsenic in $\mathrm{mg} \mathrm{kg}^{-1}$ level, checked by ICP-MS. This concentration of solid, dried alginate is far from the toxic level, stated for solid waste even for agricultural soils.

\subsection{Design of apparatus}

The first unit, the pH-adjuster contain a barrel with volume of $100 \mathrm{dm}^{3}$. That volume supplies 5 gel columns to remove the arsenate. The barrel is connected with the acidifying vessel remote by liquid level controller, the $\mathrm{pH}$-meter for regulation of $\mathrm{pH}$ and the electrical stirrer. The $\mathrm{pH}$-adjusted water is pushing through the gel column by the membrane pump, controlled by the flow meter, keeping the injected water on the column for $30 \mathrm{~min}$ as critical time for reaching the best remote of the arsenic. The puffer vessel collects the cleaned water after each column unit. The third part of the equipment is the same as the first one, but here the $\mathrm{pH}$ will be readjusted up to 7 and contains an additional vessel for adjusting the Selenate to the water. The equipment will be constructed in a module form for easy transport and installation. The connection of pipes and columns are flexible and have quick release clamps, as well as all electrical power- and remote cables have quick coupler for quick and easy maintenance.

\subsection{Remote, automation and communication}

The block scheme shows the remote and automated system with extension of communication in Fig1. The remote and automation based on PLCETHERNET Programmable Fieldbus Controller. The system is able to communicate through the Internet.

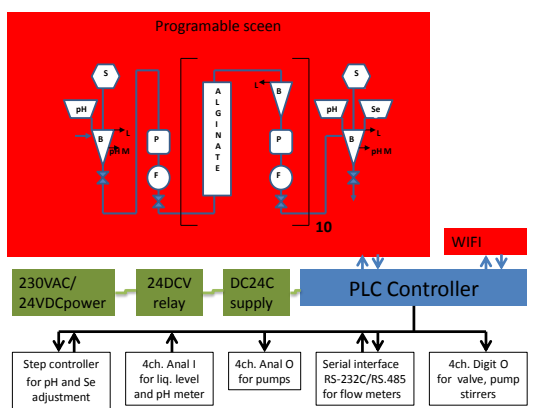

Figure 1. Block scheme of automation and communication of the arsenic remover equipment, where " $\mathrm{pH}^{\prime}$ ' is the $\mathrm{pH}$ adjuster, "S" is stirrer, " $\mathrm{B}$ " is buffer barrel, " $\mathrm{P}$ " is pump, " $\mathrm{F}$ " is flow meter, "pH M" is pH meter, "L" is liquid level sensor, "Se" is selenate adjuster

\section{CONCLUSIONS}

The automated equipment in semi-industrial scale is planning to build up for furthermore study to collect the necessary data for the industrial size instrument. The modified calcium alginate is the chemical agent, which easy to produce and environmental friendly compound, fulfilling the requirements for "green chemicals". The apparatus operate itself, communicate even through satellite, produce Arsenic clean water with cost in $4 \mathrm{USD} / \mathrm{m}^{3}$.

\section{ACKNOWLEDGEMENTS}

The authors thank the Hungarian-Indian bilateral cooperation for the financial support. The contract number is TéT-13-DST-1-2013-0015.

\section{REFERENCES}

Banerjee, A., Nayak, D. \& Lahiri. S. 2007. A new method of synthesis of iron doped calcium alginate beads and determination of iron content by radiometric method, Biochemical Engineering 33:260-262

Banerjee, A., Nayak, D. \& Lahiri. S. 2007. Speciationdependent studies on removal of arsenic by iron-doped calcium alginate beads, Applied Radiation and Isotopes 65: 769-775

Min, J.H. \& Hering, J.G. 1998. Arsenate sorption by Fe(III)doped alginate gel. Water Research 32(5): 1544-1552; US Patent 6,203,709 B1. 20.03.2001 